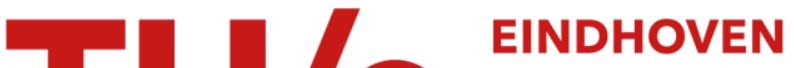

\section{Smoothed particle hydrodynamics simulations of flow separation at bends}

\section{Citation for published version (APA):}

Hou, Q., Kruisbrink, A. C. H., Pearce, F. R., Tijsseling, A. S., \& Yue, T. (2013). Smoothed particle hydrodynamics simulations of flow separation at bends. (CASA-report; Vol. 1333). Technische Universiteit Eindhoven.

\section{Document status and date:}

Published: 01/01/2013

\section{Document Version:}

Publisher's PDF, also known as Version of Record (includes final page, issue and volume numbers)

\section{Please check the document version of this publication:}

- A submitted manuscript is the version of the article upon submission and before peer-review. There can be important differences between the submitted version and the official published version of record. People interested in the research are advised to contact the author for the final version of the publication, or visit the $\mathrm{DOI}$ to the publisher's website.

- The final author version and the galley proof are versions of the publication after peer review.

- The final published version features the final layout of the paper including the volume, issue and page numbers.

Link to publication

\section{General rights}

Copyright and moral rights for the publications made accessible in the public portal are retained by the authors and/or other copyright owners and it is a condition of accessing publications that users recognise and abide by the legal requirements associated with these rights.

- Users may download and print one copy of any publication from the public portal for the purpose of private study or research.

- You may not further distribute the material or use it for any profit-making activity or commercial gain

- You may freely distribute the URL identifying the publication in the public portal.

If the publication is distributed under the terms of Article 25fa of the Dutch Copyright Act, indicated by the "Taverne" license above, please follow below link for the End User Agreement:

www.tue.nl/taverne

Take down policy

If you believe that this document breaches copyright please contact us at:

openaccess@tue.nl

providing details and we will investigate your claim. 


\section{EINDHOVEN UNIVERSITY OF TECHNOLOGY}

Department of Mathematics and Computer Science

CASA-Report I3-33

December 2013

Smoothed particle hydrodynamics simulations of flow separation at bends

by

Q. Hou, A.C.H. Kruisbrink, F.R. Pearce, A.S. Tijsseling, T. Yue

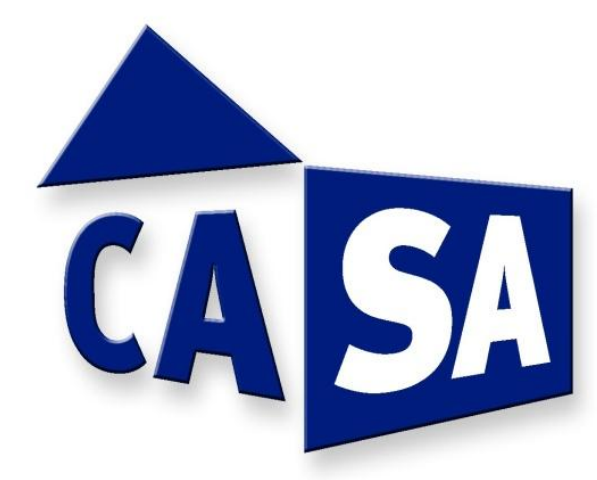

Centre for Analysis, Scientific computing and Applications

Department of Mathematics and Computer Science

Eindhoven University of Technology

P.O. Box 513

5600 MB Eindhoven, The Netherlands

ISSN: 0926-4507 



\title{
Smoothed particle hydrodynamics simulations of flow separation at bends
}

\author{
Q. Hou ${ }^{\text {a, }}$, A.C.H. Kruisbrink ${ }^{\text {b }}$, F.R. Pearce ${ }^{\mathrm{c}}$, A.S. Tijsseling ${ }^{\mathrm{a}}$, T. Yue ${ }^{\mathrm{c}}$ \\ a Department of Mathematics and Computer Science, Eindhoven University of Technology, Eindhoven, The Netherlands \\ b School of Mechanical, Materials and Manufacturing Engineering, The University of Nottingham, Nottingham, UK \\ ${ }^{\mathrm{c}}$ School of Physics and Astronomy, The University of Nottingham, Nottingham, UK
}

\begin{abstract}
The separated flow in two-dimensional bends is numerically simulated for a right-angled bend with different ratios of the channel widths and for a symmetric bend with different turning angles. Unlike the potential flow solutions that have several restrictive assumptions, the Euler equations are directly solved herein by the smoothed particle hydrodynamics (SPH) method, which is a Lagrangian approach without a mesh. The coefficient of flow contraction is obtained in terms of the ratio of the channel widths and the turning angle. The velocity field and pressure distribution in a right-angled bend are calculated. The shape of the free streamlines for a symmetric bend with several turning angles is obtained. The numerical steady-state results are validated against available theoretical solutions. The computed velocity on the free streamline is consistent with Kirchhoff's theory.
\end{abstract}

Keywords: SPH, flow separation, channel bend, free streamline, flow contraction coefficient

\section{Introduction}

Flow separation occurring in piping systems has received much attention because it determines the energy losses, pressure coefficients, flow contraction coefficients and forces on components such as pipes, valves, tees and bends. When a fluid passes a bend, it is likely to separate from the inner corner (see Fig. 1). The size of the separation void depends on the Reynolds number of the flow and the geometry of the bend. To model the flow separation problem, there are two possible ways to go. One is to use potential flow theory and the other is to solve numerically the full Navier-Stokes equations. Although the energy losses resulting from separation cannot be directly predicted by potential flow theory, a good estimate of the size of the separation region, the velocity gradients and the pressure distribution can be obtained. The potential flow solution usually agrees well with that of a high Reynolds flow. One can predict the energy dissipation resulting from flow separation through solving the Navier-Stokes equations. Such solutions can be quite difficult to obtain because of turbulence and because the separation streamline is not known in advance. Therefore, only potential flow theory applied to flow separation in bends is considered herein and briefly reviewed below.

\footnotetext{
${ }^{1}$ Correspondence to: School of Computer Science and Technology, Tianjin University, Tianjin 300072, China. E-mail: darcy.hou@gmail.com
} 


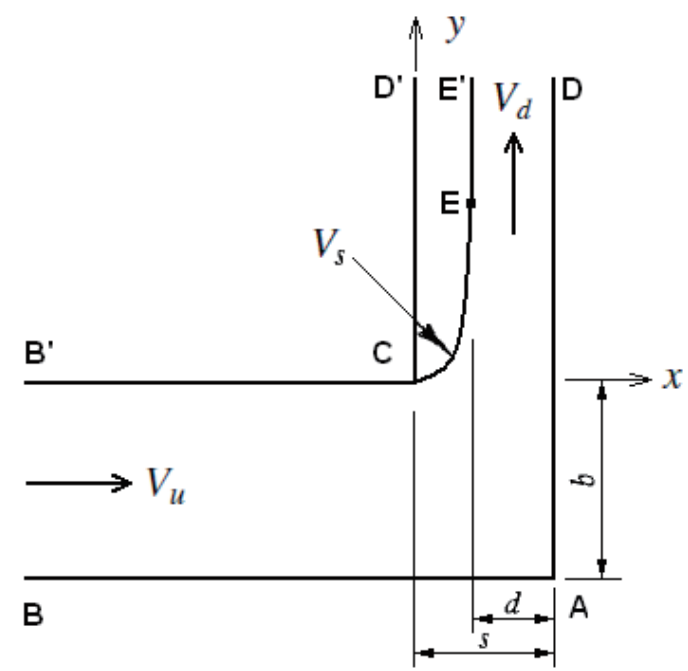

Figure 1: Definition sketch of separated flow in a right-angled bend.

Using conformal mapping and Roshko's free-streamline theory [1], Lichtarowicz and Markland [2] solved the potential flow round a right-angled elbow for two different ratios of the channel widths $R_{b}:=s / b$ (see Fig. 1). In Roshko's model, separation is introduced through a free streamline that divides the flow in the bend into two regions: 1) the main flow where the velocity is continuous and possesses a potential, and 2) a secondary region extending theoretically to infinity. The separated free streamline is assumed to start from point $C$, curve gradually until its direction is that of the secondary flow and then remain straight and parallel to the wall. The position of the conjuncture point $E$ and the velocity along the streamline $V_{s}$ are functions of the ratio of the velocity at the separation point to the velocity far downstream. By mapping the physical plane onto a hodograph domain, Mankbadi and Zaki [3] studied the flow patterns in symmetric and asymmetric bends with various turning angles $\beta$ (see Fig. 2). In contrast to Lichtarowicz and Markland [2], Kirchhoff's free-streamline theory has been used in [3]. In Kirchhoff's theory, the curved free streamline starts from point $C$ and asymptotically extends to infinity. That is, there is no second flow region as in Roshko's model. The velocity on the free streamline $C E^{\prime}$ is assumed to remain constant and is equal to that of the downstream flow, i.e. $V_{s}=V_{d}$. The hodograph transformation method used in $[3,4]$ is efficient for two-dimensional and axisymmetric problems [4, 5]. According to Hassenpflug [6], when the liquid is at very high Reynolds numbers and the region adjacent to the free streamline is gas, the model is a good description of the actual physical flow. However, the solution procedure of the indirect hodograph method is rather restrictive [4], and in practice it is difficult to impose the boundary conditions as assumed in the theory [6].

During the early developments (1980s) of the smoothed particle hydrodynamics (SPH) method, it was mainly applied to compressible astrophysical flows [7]. Today SPH is used to model the collapse and formulation of galaxies, coalescence of black holes with neutron stars, detonations in white dwarfs and even the evolution of the universe [8, 9]. At the beginning of the 1990s, SPH was extended to model high velocity impact problems of solids [10] and incompressible free-surface flows [11]. Now SPH is used to simulate a vast range of fluid dynamic problems as 


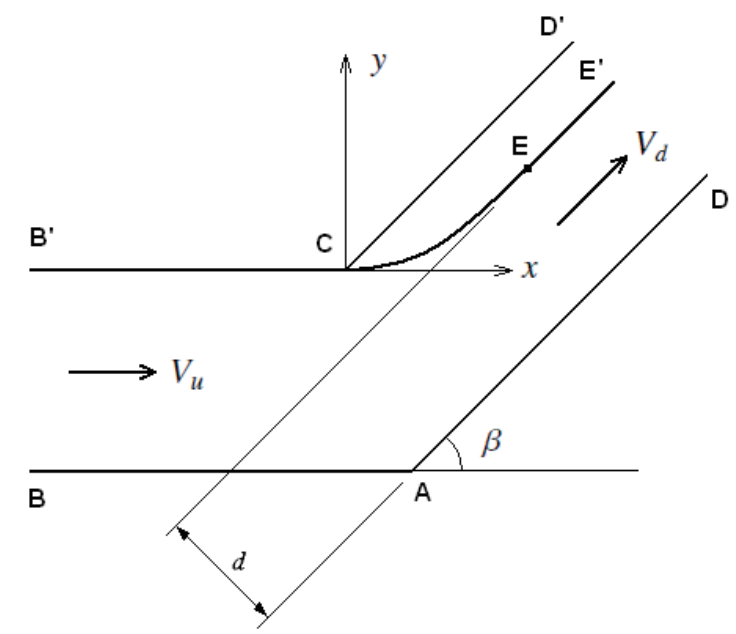

Figure 2: Definition sketch of separated flow in a symmetric bend with turning angle $\beta$.

shown in the recent reviews $[12,13]$. However, there is not much published work on SPH applications to pipe related flows, which are generally treated as 1D problems. For 1D flows, SPH has less advantage over traditional mesh-based methods than for 2D and 3D flows. The most studied case is the shock tube problem [7, 8, 14]. Lastiwka et al. [15] used SPH for 1D compressible nozzle flows. Recently, it was successfully employed to model rapid pipe filling [16], water hammer [17] and slug impact [18]. The SPH simulations of the 2D impinging jet on an inclined wall [19, 20, 21] have close relationship with the problem considered herein.

The SPH method is applied in this paper to study flow separation at bends with various aspect ratios $R_{b}$ (Fig. 1) and turning angles $\beta$ (Fig. 2). The SPH approximations discretize the spatial derivatives through particles that move with the flow. The flow properties carried by the moving particles are computed from interaction with their neighbouring particles. A priori connectivity between the particles is not required. As free surfaces are naturally captured and represented by moving particles, the dedicated surface tracking techniques encountered in traditional mesh-based methods are not needed.

The paper is organized as follows. Section 2 presents the discrete SPH equations describing inviscid flow. The numerical treatment of various boundary conditions is described and several important aspects of the numerical implementation are discussed. In Section 3, the obtained numerical results are compared with theoretical results. Concluding remarks are given in Section 4.

\section{SPH fluid dynamics}

The SPH equations describe the evolution of the flow and the motion of material points that are referred to as particles. Each particle, e.g. particle labeled $a$, carries a constant mass $m_{a}$, and a time-dependent density $\rho_{a}$, pressure $p_{a}$ and velocity $\mathbf{v}_{a}$. The particle changes its position $\mathbf{r}_{a}$ according to the flow velocity. In contrast to traditional Eulerian methods, SPH is a Lagrangian particle solver where the particle connectivity evolves with time and needs to 
be determined by a particle search. The SPH equations for a compressible gas are described in [7]. Herein the SPH equations for a weakly compressible inviscid fluid are presented. More details can be found in the recent review [13].

\subsection{Discrete SPH equations}

The definition sketches of a separated flow inside a right-angled bend and a symmetric bend with an arbitrary turning angle $\beta$ are shown in Figs. 1 and 2, respectively. The fluid flows in the bend from the inlet $B B^{\prime}$ to the outlet $D D^{\prime}$. Two outer walls $B A$ and $A D$, and two inner walls $B^{\prime} C$ and $C D^{\prime}$, form the fixed boundaries. At point $C$ the flow separates from the wall and follows the curved free streamline $C E^{\prime}$. The flow is assumed to be two-dimensional and the modeled fluid is weakly compressible and inviscid. There is no gravity, so that Figs. 1 and 2 are top views. The problem is governed by the Euler equations, which in discrete SPH form read [11, 13]:

$$
\begin{gathered}
\frac{D \rho_{a}}{D t}=\sum_{b} m_{b} \mathbf{v}_{a b} \cdot \nabla_{a} W_{a b}, \\
\frac{D \mathbf{v}_{a}}{D t}=-\sum_{b} m_{b}\left(\frac{p_{a}}{\rho_{a}^{2}}+\frac{p_{b}}{\rho_{b}^{2}}+\Pi_{a b}\right) \nabla_{a} W_{a b} .
\end{gathered}
$$

In the discrete SPH continuity equation (1), subscripts $a$ and $b$ denote a reference particle $a$ and its neighbours $b$; $\mathbf{v}_{a b}:=\mathbf{v}_{a}-\mathbf{v}_{b} ; W_{a b}:=W\left(\mathbf{r}_{a}-\mathbf{r}_{b}, h\right)$ is the kernel (see Section 2.3); $\nabla_{a} W_{a b}$ is the gradient of the kernel taken with respect to the position of particle $a$. The smoothing length $h$ is a size scale of the kernel support and determines the degree that a particle interacts with its neighbours. In the discrete SPH momentum equation (2), $\Pi_{a b}$ is an artificial viscous term that has the general form

$$
\Pi_{a b}:=\frac{-\alpha c_{0} h}{\bar{\rho}_{a b}\left(r_{a b}^{2}+0.01 h^{2}\right)} \min \left(\mathbf{v}_{a b} \cdot \mathbf{r}_{a b}, 0\right),
$$

in which $c_{0}$ is the speed of sound, $\mathbf{r}_{a b}:=\mathbf{r}_{a}-\mathbf{r}_{b}, r_{a b}:=\left|\mathbf{r}_{a b}\right|, \bar{\rho}_{a b}:=\left(\rho_{a}+\rho_{b}\right) / 2$, and $\alpha$ is a problem-dependent constant $[7,11]$. Here we take $\alpha=0.1$ as proposed in [20] for free-surface flows. This artificial term produces a shear and bulk viscosity in the flow.

To close the system, the gauge pressure of particle $a$ correlates to its density by an equation of state [22],

$$
p_{a}=c_{0}^{2}\left(\rho_{a}-\rho_{0}\right),
$$

where $\rho_{0}$ is the fluid density at a reference pressure. The value of $c_{0}$ needs some care as explained below. For hydraulic and acoustic pressure variations, the relative density variation $\delta \rho / \rho$ is proportional to $\mathrm{Ma}^{2}$ [11] and $\mathrm{Ma}$, respectively, where $\mathrm{Ma}:=V / c_{0}$ is the Mach number and $V$ is a typical convective velocity. Since $V$ is generally two or three orders of magnitude smaller than $c_{0}, \delta \rho / \rho$ is extremely small. In SPH, incompressible and weakly compressible fluids are approximated by an artificial fluid which is much more compressible. The relative density variation $\delta \rho / \rho$ is generally taken to be about $1 \%$ by using an artificial speed of sound $c_{0}$. After the estimation of a typical velocity $V$, e.g. the inflow velocity in this study, a suitable choice of $c_{0}$ produces the desired density variation of about $1 \%$. 


\subsection{Boundary conditions}

For the problems considered herein, four types of boundary conditions need to be numerically treated. They are the free-slip wall, free surface, inlet and outlet. The mathematical statements of these boundary conditions can be found in e.g. [21, 23]. Here we briefly discuss their treatment in SPH; further details can be found in [21].

There are several methods to deal with the free-slip wall condition. The wall particle method [11] is easy to implement and useful for irregular boundaries. It used to be popular but is rarely used now, because nonphysical shear stresses may occur. The fixed ghost particle method proposed by Morris et al. [22] is another choice. It was mainly used for no-slip boundary conditions, and its recent extension to free-slip walls using interpolation and extrapolation techniques is detailed in [24]. Based on an idea similar to the fixed ghost particle method, Adami et al. [25] recently proposed a new approach. Another common way to enforce the free-slip condition is to use a local mirroring of the fluid particles onto the other side of the solid boundary [26, 27]. At each time step an image of the flow is generated. The thickness of the mirror particle layer is slightly larger than the kernel radius to ensure that all kernels are complete. To exactly satisfy the free-slip condition, the tangent velocity is the same as that of the fluid particle, whilst the normal velocity is in opposite direction [21]. The mirror particle approach is used in this study; its efficiency has been underlined by Monaghan [13].

As seen in Figs. 1 and 2, when two straight walls join at some point, a corner (geometric singularity) is formed. To complete the support of the kernel associated with particles close to the corner, additional treatment is needed. For consistency, a similar idea as the mirror particle approach is applied. The empty space which is left behind the two walls is filled with corner mirror particles by applying a point-symmetry to the fluid particles near the corner. The treatment of geometric singularities with the fixed ghost particle method is described in [24].

On the free surface two conditions need to be satisfied. The kinematic condition implies that a particle originally on the surface will remain on it. This is naturally satisfied by the Lagrangian particle movement. The dynamic condition $(p=0)$ is automatically satisfied too due to the SPH formulation of the continuity equation and spatial derivative $[21,28]$. The automatic enforcement of the free surface conditions is an inherent advantage of SPH over traditional mesh-based methods.

The boundary conditions at the inlet and outlet are enforced by using the image particle approach [15, 20, 29]. An inflow buffer block is placed ahead of the planar inlet boundary. The thickness of the block is $2.5 h$, which is slightly larger than the support radius of the kernel. In the inlet block, there are predefined image particles (referred to as inlet particles). An inlet particle moves with its given velocity. After crossing the inlet and entering the fluid domain, it becomes a fluid particle and the flow field associated with it will evolve from the next step on. A new inlet particle is created at the upstream end of the inlet buffer block. Similarly, an outflow buffer block is placed behind the outlet boundary to ensure that the fluid flows out of the fluid domain freely. When a fluid particle leaves the fluid domain and enters the outlet block, it becomes an outlet particle whose properties will not change. It will be deleted after leaving the outflow buffer block at its downstream end. This is different from the non-reflecting SPH boundary proposed by Lastiwka et al. [15], in which an extrapolation is necessary to calculate the flow fields in the buffer blocks. The inflow 


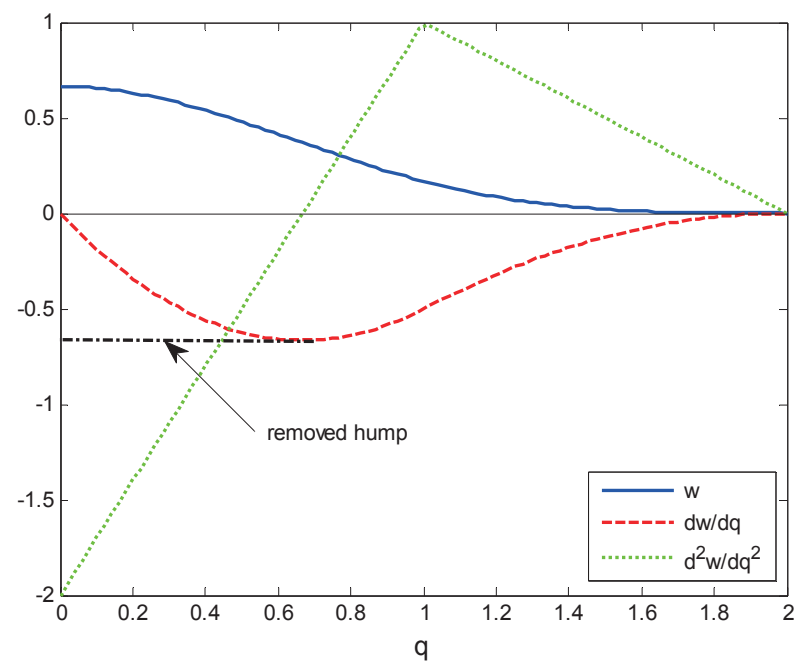

Figure 3: The cubic spline kernel $W(q)$ and its derivatives.

and outflow sections should be far enough from the bend to avoid any influence from the creation of inlet and removal of outlet particles [21].

\subsection{Kernel and its gradient}

The use of different kernels $W_{a b}$ with different $h$ is the SPH analogue of using different stencils in finite-difference methods [30]. Fulk and Quinn [31] analyzed 20 different SPH kernels and concluded that the bell-shaped kernel$\mathrm{s}$ usually perform better than other shapes. The following bell-shaped cubic-spline kernel has been proven to be computationally accurate $[7,31]$ :

$$
W(q):=G \begin{cases}1-1.5 q^{2}+0.75 q^{3}, & 0 \leqslant q<1, \\ 0.25(2-q)^{3}, & 1 \leqslant q<2, \\ 0, & q \geqslant 2,\end{cases}
$$

where $q:=r_{a b} / h$ and the normalizing coefficient $G$ is $10 /(7 h \pi)$ for two-dimensional problems. The cubic-spline kernel and its derivatives are shown in Fig. 3.

For free-surface flows, the smoothing length is generally taken as $h=\eta d_{0}$ where $\eta=1.1 \sim 1.33[11,20,32]$ and $d_{0}$ is the initial particle spacing (particles are placed on a square lattice). Here we take $\eta=1.33$ as in [20]. To avoid possible "pairing instability" resulting from a relatively large $\eta$ [14], the "hum" in the kernel gradient is removed by simply making the kernel gradient constant for $q<2 / 3$ [33] (see Fig. 3). As discussed by Price [14], "Whilst removing the hump cures the pairing instability, one should be careful about employing such a gradient in practice since the kernel gradient is no longer exactly normalized. The pairing instability is the main reason one cannot simply stretch the cubic spline to large neighbour numbers in order to obtain convergence." 


\subsection{Time stepping}

Starting from an initial distribution ( $\mathbf{r}_{a}$ ) of particles with given mass $m_{a}$ (constant in time), densities $\rho_{a}$ and velocities $\mathbf{v}_{a}$, the basic equations (1), (2) are solved at each time step for each particle. For time integration Euler's forward method is used herein, which is first-order accurate, fully explicit, and conditionally stable. A recommended time step size satisfying the Courant-Friedrichs-Lewy (CFL) criterion is [22]:

$$
\Delta t \leq C_{C F L} \frac{h}{c_{0}+|V|}
$$

where $C_{C F L}$ is a constant between 0 and 1 . With $\mathrm{Ma}=|V| / c_{0}$ the CFL condition (6) can be rewritten as

$$
\Delta t \leq C \frac{d_{0}}{c_{0}}
$$

where the constant $C=\eta C_{C F L} /(1+\mathrm{Ma})$ is taken as 0.2 herein. In addition, a time-step constraint related to acceleration has to be satisfied by [34]:

$$
\Delta t \leq \frac{1}{4} \min _{b}\left(\sqrt{\frac{h}{|\mathbf{a}|_{b}}}\right),
$$

where $|\mathbf{a}|_{b}=\left|D \mathbf{v}_{b} / D t\right|$ is the magnitude of particle acceleration and the minimum is over all particles.

To efficiently find and access neighbouring particles at each time step, the link-list algorithm with an optimized cell size [35] is used.

\section{Numerical results}

Two series of flow separation problems in rectangular channels are simulated. The right-angled elbow shown in Fig. 1 is considered first. The width of the upstream channel is fixed at $b=1 \mathrm{~m}$, whilst the downstream channel width $s$ is varied and related to $b$ by the ratio of channel widths $R_{b}$. The length of the inner walls $B^{\prime} C$ and $C D^{\prime}$ is 2 m. The length of the outer wall $A D$ is $3 \mathrm{~m}$ and the length of $B A$ depends on the given value of $R_{b}$. The second series of simulations concern flow separation at a symmetric bend $\left(R_{b}=1\right)$ with various turning angles $\beta$ (see Fig. 2). The lengths of $B^{\prime} C$ and $C D^{\prime}$ and the channel width $b$ are the same as above. The chosen lengths of the outer walls depend on the given turning angle $\beta$.

In the SPH setup, there are uniformly distributed fluid particles upstream of the bend $(X<0)$ and inlet particles in the inflow section with particle spacing $d_{0}=0.05 \mathrm{~m}\left(b / d_{0}=20\right)$. The pressure is zero and the velocity components are $V_{u}=V_{x}=1 \mathrm{~m} / \mathrm{s}$ and $V_{y}=0$. The artificial speed of sound is taken as $c_{0}=15 \mathrm{~m} / \mathrm{s}$, which gives a sufficiently low Mach number. The time step for all cases considered herein is fixed at $0.0001 \mathrm{~s}$, which is small enough to satisfy the stability conditions (7) and (8). When the kinetic energy of all particles in the computational domain becomes constant (relative difference between two time steps is less than a given tolerance), the simulation is stopped and assumed to have reached its steady state. The spatial coordinates are scaled by the upstream channel width: $X=x / b$ and $Y=y / b$. 


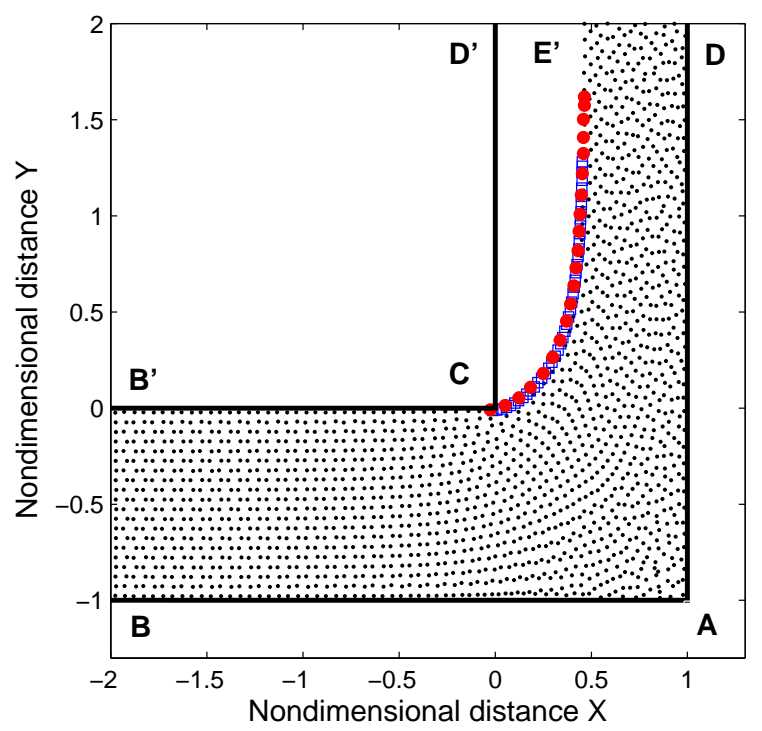

Figure 4: Particle paths and theoretical free-streamline in a right-angled bend. Dots - SPH particles; open squares - L \& M [2]; filled circles - Chu [4].

For a right-angled elbow with $R_{b}=1$, the SPH solution is shown in Fig. 4, where the outer particle layer represents the free streamline. The solutions from potential flow theory are also presented for comparison. The computed freestreamline matches the theoretical solutions very well. The agreement of the current results with the solution of Lichtarowicz and Markland [2] is slightly better than with that of Chu [4]. Some error may be present in the results extracted from Chu [4], as his coordinate information is incomplete. The cross-sectional averaged outlet velocity at $Y=2$ is $V_{d}=1.89 \mathrm{~m} / \mathrm{s}$, which is slightly smaller than the prediction $\left(V_{d}=1.90 \mathrm{~m} / \mathrm{s}\right)$ of Chu [4]. The slip velocity distribution along wall $A D$, scaled by $V_{d}$, is shown in Fig. 5. The SPH prediction is consistent with the theoretical solution of [4]. The particle velocity along the free streamline $C E^{\prime}$ is constant at a value close to the averaged outlet velocity $V_{d}$. This is consistent with Kirchhoff's free-streamline theory.

To show the numerical convergence, simulations with coarser $\left(b / d_{0}=10\right)$ and finer $\left(b / d_{0}=40\right)$ initial particle spacings were performed and the results are also presented in Fig. 5. The convergence is evident and the result with the highest resolution $\left(b / d_{0}=40\right)$ has the best agreement with the theory. The convergence rate is of first order [21], which is consistent with the results of other researchers [36, 37, 38]. Since the SPH solution with $b / d_{0}=20$ is sufficiently accurate for illustration purposes, the following results are obtained with this particle resolution.

The distribution of the pressure coefficient $C_{p}$ is shown in Fig. 6, together with the results of Lichtarowicz and Markland [2] and Mankbadi and Zaki [3]. The pressure coefficient is defined as $C_{p}:=p /\left(\rho V_{d}^{2} / 2\right)$, in which $p$ is the pressure along the outer wall $A D$. There are two ways to determine the SPH pressure $p$ at steady state. One way is to derive it from the velocity distribution through the Bernoulli theorem, which is entirely consistent with the steady Euler equations. In this approach, the velocity along the wall is calculated first by interpolating the particle velocities 


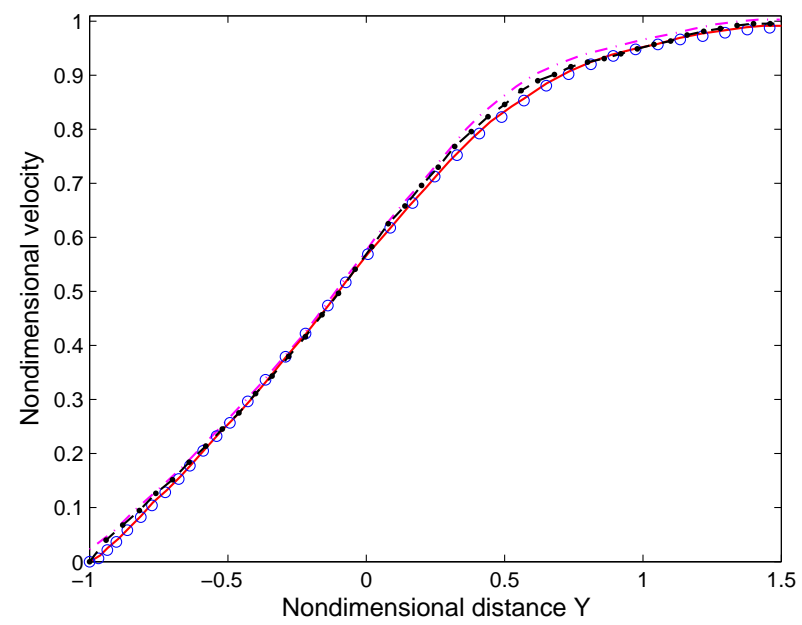

Figure 5: Velocity distribution along wall $A D$. Dashed line $-\mathrm{SPH}$ with $b / d_{0}=10$, solid line with dots $-\mathrm{SPH}$ with $b / d_{0}=20$, solid line $-\mathrm{SPH}$ with $b / d_{0}=40$; open circles - Chu [4].

as shown in Fig. 5. The pressure is then computed according to the Bernoulli equation. The other way is to directly interpolate from the particles describing the pressure field. The SPH results shown in Fig. 6 are determined by the first approach. The predicted $C_{p}$ by SPH has better agreement with the solutions of Lichtarowicz and Markland [2] than with those of Mankbadi and Zaki [3]. This is consistent with the conclusion of Chu [4], that numerical errors may have been present in the method of the latter. The directly interpolated pressure distribution (not shown here) has less satisfying agreement with the theoretical solutions because of noise in the pressure field [20, 34].

For the asymmetric case $\left(R_{b}:=s / b \neq 1\right)$, four steady-state flow fields are displayed in Fig. 7 . For the free streamlines, there are no theoretical results available for comparison, but the computed results can be verified to some extent through the contraction coefficient $C_{c}:=d / b$ (see Fig. 1) as shown in Table 1 . Note that the flow width $D E^{\prime}$ at the outflow section has a small variation in time due to particle fluctuations. Consequently, the evaluation of $C_{c}$ involves averaging over a certain time interval at steady - but slightly fluctuating - state. The calculated contraction coefficients agree very well with the theoretical ones. The maximum relative error is less than 1.5 percent.

Table 1: Values of contraction coefficients $C_{c}$ for different ratios of channel widths $R_{b}$.

\begin{tabular}{lllllllllllll}
\hline$R_{b}$ (Ratio) & 0 & 0.5 & 0.6 & 0.7 & 0.8 & 0.9 & 1.0 & 1.2 & 1.3 & 1.5 & 2.0 & $\infty$ \\
\hline L \& M [2] & $0.611^{*}$ & 0.584 & 0.573 & 0.560 & 0.551 & 0.537 & 0.526 & 0.500 & $-^{\dagger}$ & $-^{\dagger}$ & $-^{\dagger}$ & $1.0^{\ddagger}$ \\
Present & $-^{\dagger}$ & 0.59 & 0.58 & 0.56 & 0.55 & 0.53 & 0.53 & 0.50 & 0.63 & 0.69 & 0.81 & $-^{\dagger}$ \\
\hline
\end{tabular}

$\dagger$ no solution available; ${ }^{\star}$ analytical solution given in [20, 39]; ${ }^{*}$ analytical solution given in [2].

As shown in Table 1 , the contraction coefficient $C_{c}$ decreases as $R_{b}$ increases up to $R_{b}=1.2$. When $R_{b}$ becomes so large that the separation point $C$ has no effect on the downstream flow, the current setup will be that of half of a 


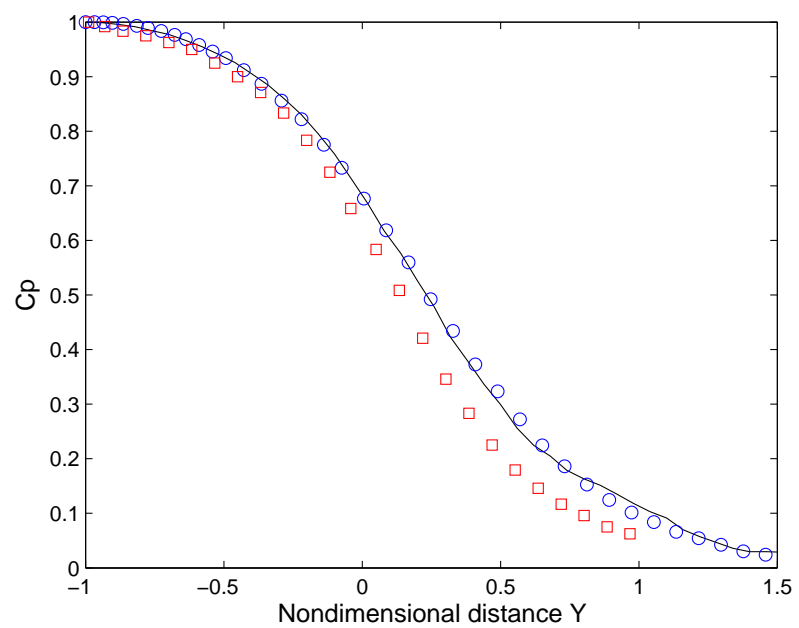

Figure 6: Nondimensional pressure along wall $A D$. Solid line - present SPH; open squares - L \& M [2]; open circles - M \& Z [3].
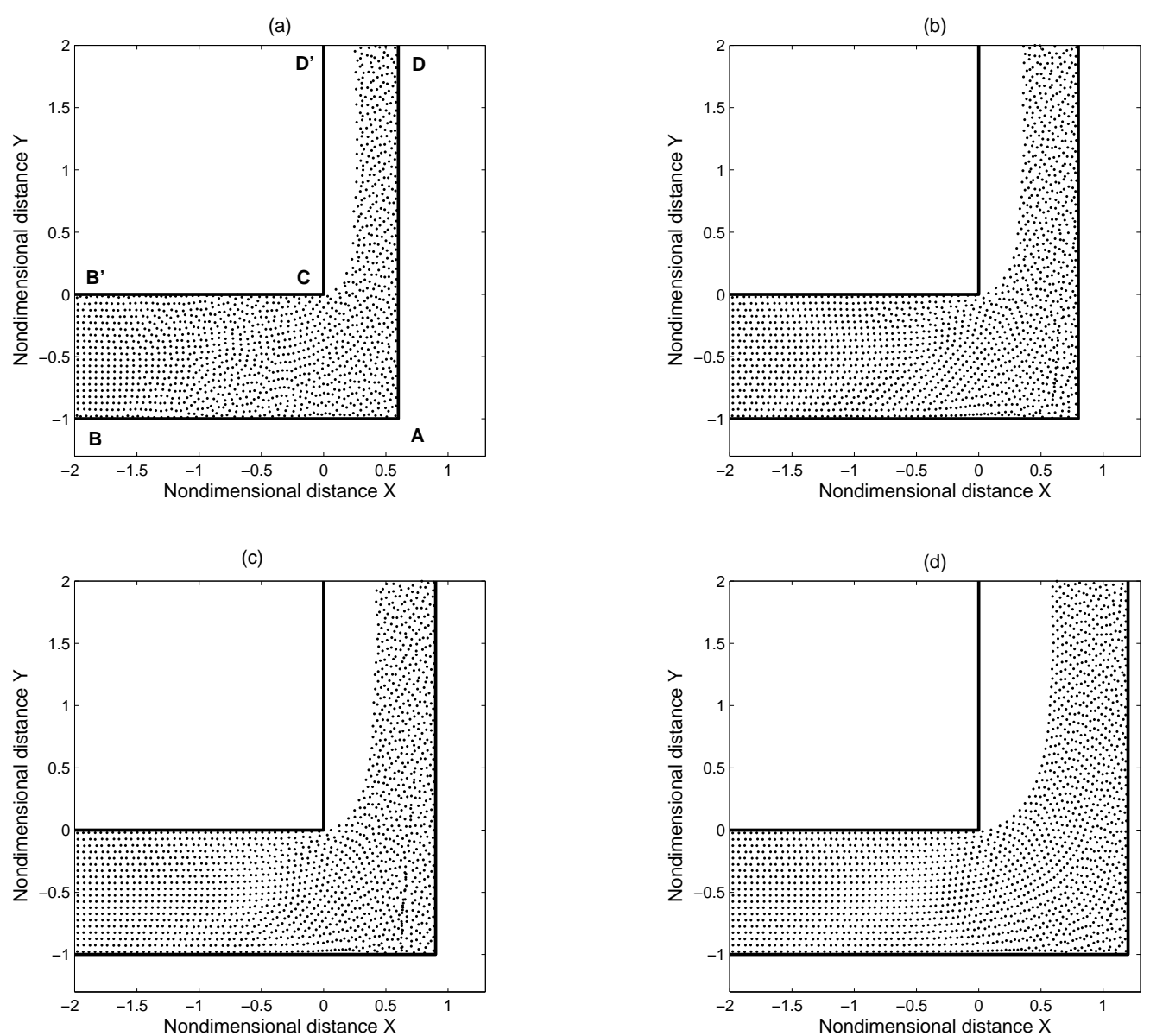

Figure 7: Flow in a right-angled bend for different ratios of channel widths: (a) $R_{b}=0.6$, (b) $R_{b}=0.8$, (c) $R_{b}=0.9$ and (d) $R_{b}=1.2$. 
jet emerging from a channel and impinging on an orthogonal plane [20,21]. That is, when $R_{b}$ becomes large enough, the bend (i.e. outer wall $A D$ ) has no effect on the upstream parallel flow. Clearly, following the same definition, the contraction coefficient for that case is 1 . That is, as $R_{b} \rightarrow \infty$ then $C_{c} \rightarrow 1$ [20,39]. As a consequence, there will be a specific $R_{b}$ (between 1.2 and 1.3) having a minimum value of $C_{c}$ (about 0.5). On the other hand, when $R_{b} \rightarrow 0$, the contraction coefficient approaches another constant 0.611 [2]. This limit case cannot be simulated easily in the present method or any other mesh-based numerical method. In fact, when $R_{b}$ equals 0.5 , the simulated flow has become so violent that the effect of the inlet location is not negligible anymore.

Typical results of the second series of simulations $\left(R_{b}=1\right.$ with different angle $\left.\beta\right)$ are shown in Fig. 8 together with results from potential flow theory. For the first three cases $\beta=15^{\circ}, 30^{\circ}$ and $45^{\circ}$, the theoretical curves of the free streamlines were not given in $[3,4]$. For the other three cases $\beta=60^{\circ}, 120^{\circ}$ and $150^{\circ}$, the numerical results agree very well with the theoretical solutions. The contraction coefficients for various turning angles are shown in Table 2. The numerical results are consistent with the theoretical predictions. The relative error is less than 1 percent.

Table 2: Values of contraction coefficients $C_{c}$ for different turning angles $\beta$.

\begin{tabular}{llllllll}
\hline$\beta\left({ }^{\circ}\right)$ & 15 & 30 & 45 & 60 & 90 & 120 & 150 \\
\hline Chu [4] & 0.893 & 0.792 & 0.701 & 0.625 & 0.528 & 0.467 & 0.434 \\
Present & 0.89 & 0.79 & 0.70 & 0.63 & 0.53 & 0.47 & 0.43 \\
\hline
\end{tabular}

To reach the final steady state, the simulation time varied from 4 seconds $\left(\beta=15^{\circ}\right)$ to 5 seconds $\left(\beta=150^{\circ}\right)$. That is, after about 45000 time-steps, the plotted final states were achieved. All the calculations were performed on a standard PC, and the computation time was between 15 and 25 minutes for one complete case. The number of fluid particles in steady state varied from $1800\left(\beta=15^{\circ}\right)$ to $2950\left(\beta=150^{\circ}\right)$. The obtained agreement can even be further improved by increasing $b / d_{0}$ (reducing the initial particle spacing $d_{0}$ ) at the expense of computational time. By halving the particle spacing, the number of fluid particles at steady state is approximately four times more, and hence the CPU time will increase four times. As shown in Fig. 8, with the increasing of $\beta$, a larger portion of the flow is affected by the outer corner of the bend, and less particles stay in smooth streamlines when rounding the bend. When the turning angle is larger than $90^{\circ}$, some fluid particles remain trapped at the outer corner (see Figs. 8e and 8f).

Based on Table 2, the estimated maximum velocity at steady state for the cases considered is $V_{\max }=V_{u} / C_{c}<2.5$ $\mathrm{m} / \mathrm{s}$. Thus a speed of sound $c_{0}=25 \mathrm{~m} / \mathrm{s}$ should guarantee the $\mathrm{SPH}$ requirement of low Mach number for all test cases. However, when $c_{0}=25 \mathrm{~m} / \mathrm{s}$ is used, during the early unsteady stage, particles penetrate through the outer corner of bends with large turning angles (e.g. $\beta=120^{\circ}$ and $150^{\circ}$ ). The reason is that the maximum velocity during the unsteady part of the numerical simulation can be much higher than $2.5 \mathrm{~m} / \mathrm{s}$. For a flow starting with a vertical front (see Fig. 9a), a large portion of it will turn downwards to the outer corner because there is no entrapped air to 
(a)

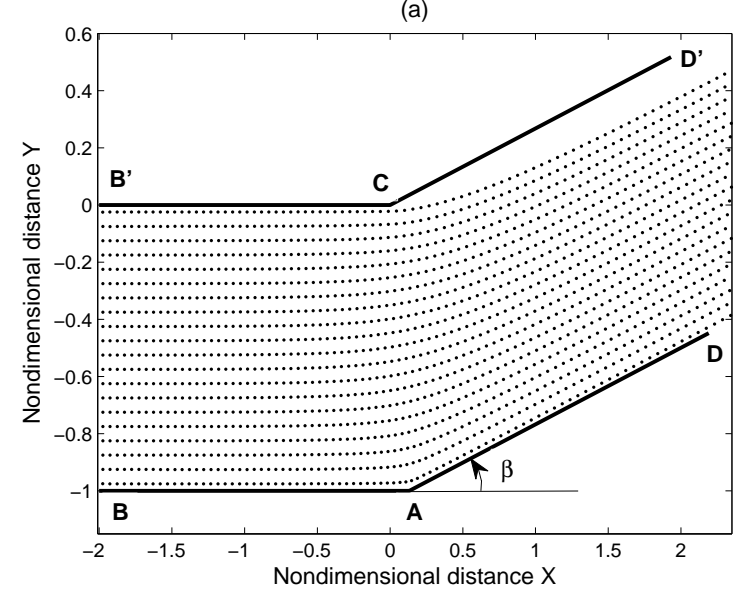

(c)

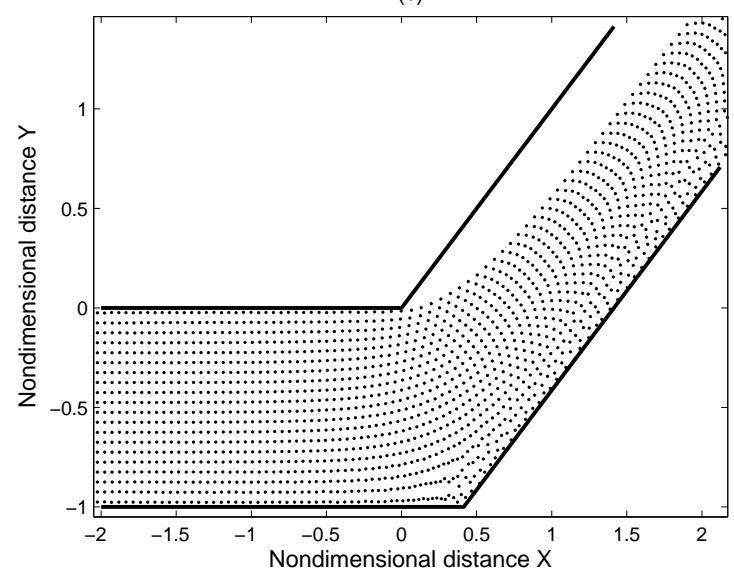

(e)

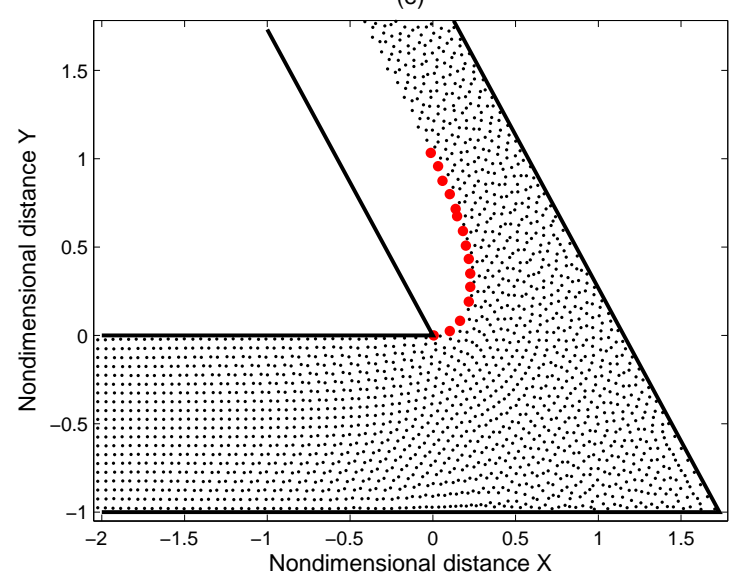

(b)

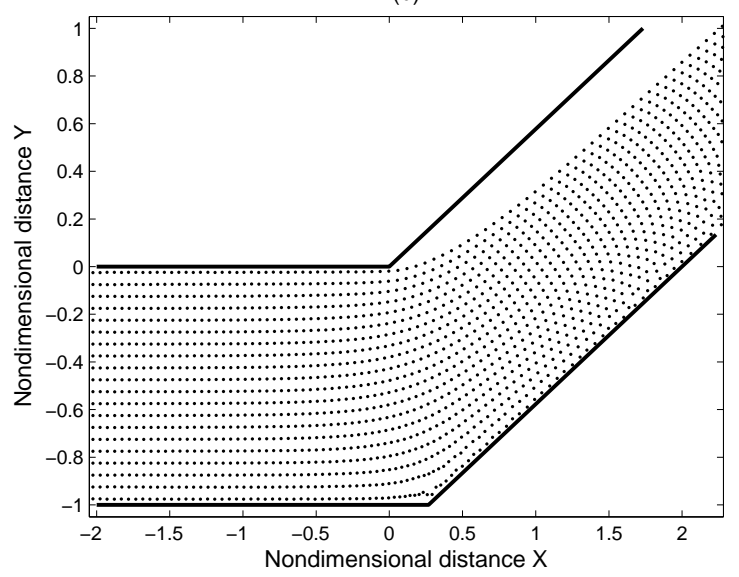

(d)

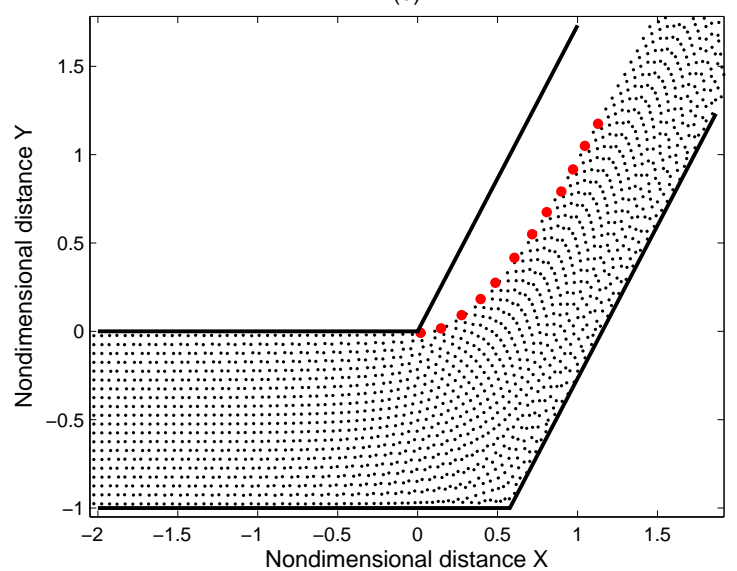

(f)

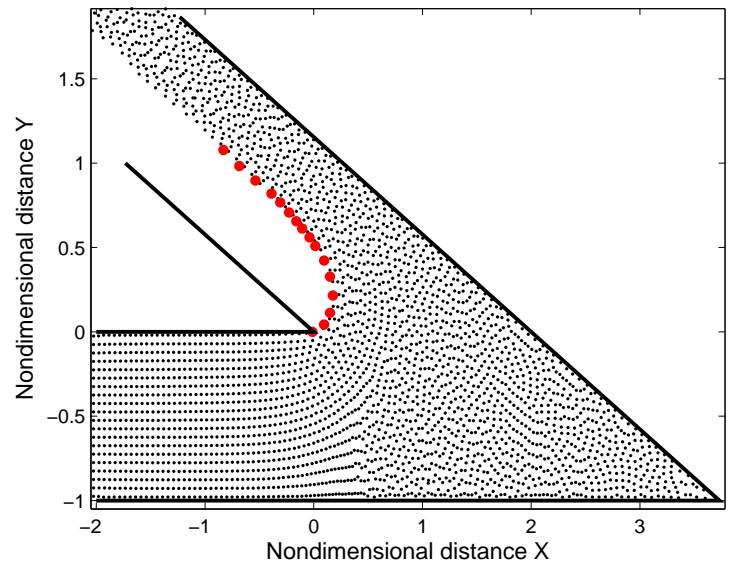

Figure 8: Flow in symmetric bends with various turning angles: (a) $\beta=15^{\circ}$, (b) $\beta=30^{\circ}$, (c) $\beta=45^{\circ}$, (d) $\beta=60^{\circ}$, (e) $\beta=120^{\circ}$ and (f) $\beta=150^{\circ}$. Dots - SPH particles; Filled circles - Chu [4] 
prevent it (see Figs. 2 and 9b). Due to conservation of volume, the local flow velocity increases and attains a high value before it arrives at the outer corner, e.g. a velocity of $9.5 \mathrm{~m} / \mathrm{s}$ in the $\beta=150^{\circ}$ case. The pressure forces exerted by mirror particles at the opposite side of the wall are not high enough to fully stop the high velocity particles going into the corner, and some particles penetrate through the geometric singularity. This artefact happens mainly during the early unsteady phase of the simulation, and fully disappears at steady state.

(a)

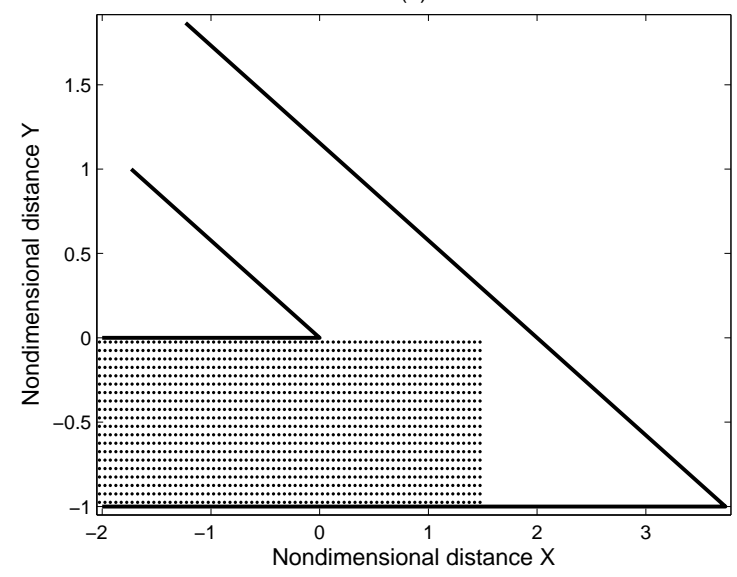

(c)

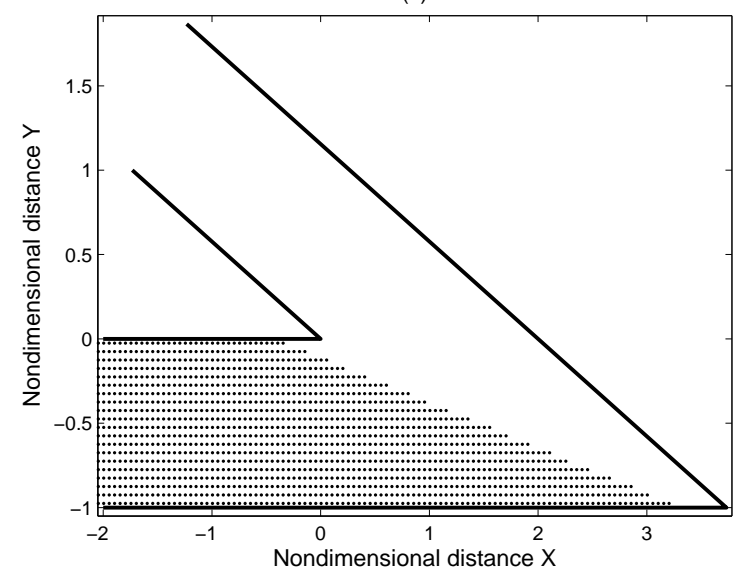

(b)

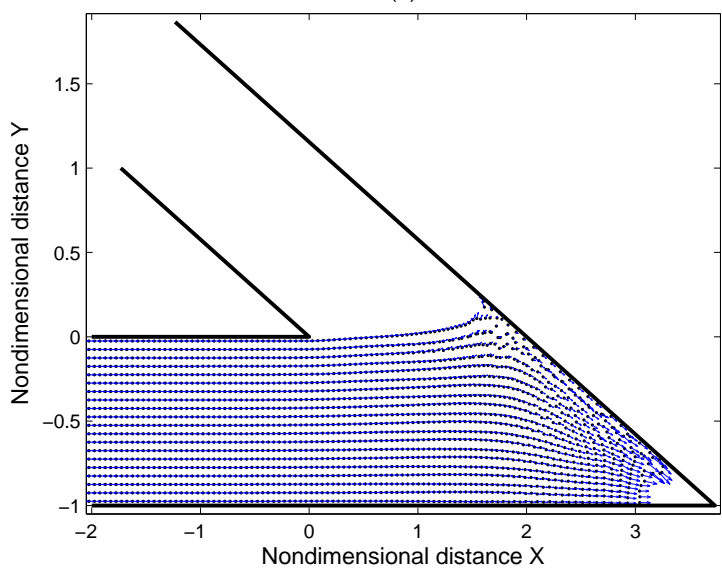

(d)

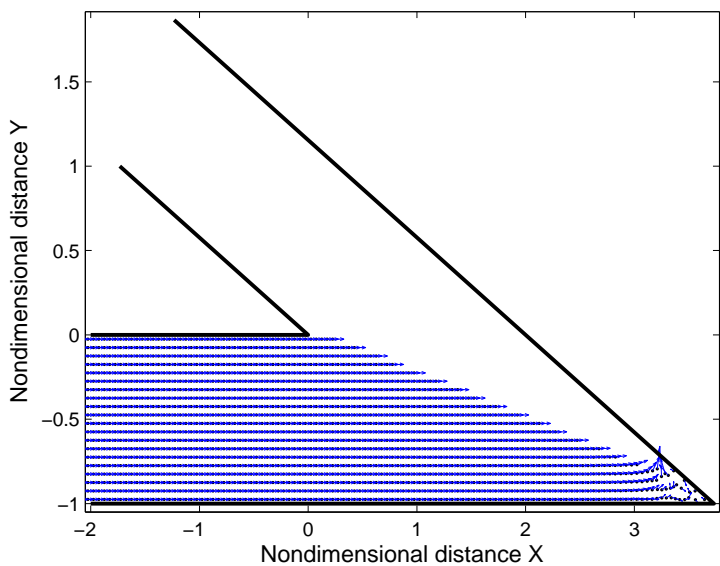

Figure 9: Sketch of possible particle penetration at the outer corner when too small $c_{0}$ is used: (a) vertical front, (b) velocity distribution, (c) wedge front and (d) velocity distribution.

To avoid early-stage particle penetration, a possible way is to use a larger $c_{0}$, i.e. $c_{0}=95 \mathrm{~m} / \mathrm{s}$, to fulfill the requirement of low Mach number. This increases the pressure forces exerted by the mirror particles. However, when $c_{0}=95 \mathrm{~m} / \mathrm{s}$ is used, the Mach number at steady state will not be within the desired range $\Delta \rho / \rho=\mathrm{Ma}^{2} \sim 1 \%$. Hence a time-dependent speed of sound should be used at the expense of one more equation that needs to be solved (see e.g. [40] for this new concept). In fact, there is a simpler way to avoid early-stage particle penetration without using a larger or time-dependent $c_{0}$ than practically desired. The initial flow is set up with a wedge front (the angle of which 


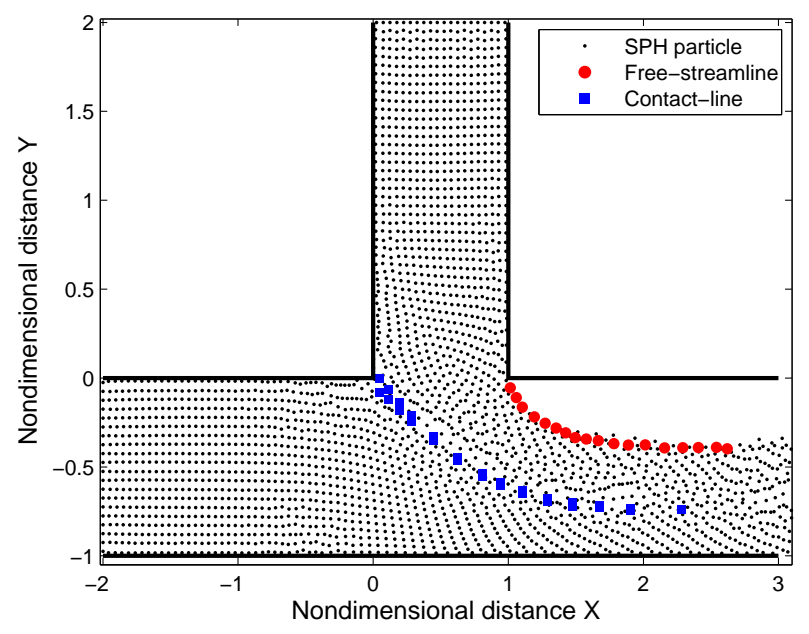

Figure 10: Flow with free-streamline and contact-line in a channel with orthogonal side branch. Dots - SPH particles; Filled circles and squares Hassenpflug [6].

is larger than $\beta$ ) as shown in Fig. 9c. The maximum velocity during the early unsteady state is now reduced to $1.4 \mathrm{~m} / \mathrm{s}$ and the particle distribution is less disordered (see Fig. 9d). Although the ultimate free-streamline profiles at steady state show no significant change, the unsteady simulation becomes smoother, and the steady state is achieved earlier.

Another practical situation is the flow in branched channels as systematically studied by Hassenpflug [6]. To demonstrate the capability of the present method to simulate flow separation in branched channels, the first example of Hassenpflug [6] consisting of two perpendicular inlets with identical flow velocities is examined here, and the results are shown in Fig. 10. To avoid double particle mirroring at the left inner corner, two orthogonal continuous walls [28] with a length of $2 h$ were used for the enforcement of the free-slip condition. It is seen that for both the free streamline and the contact line of the two inflows, the computed solutions are consistent with the analytical solution. The small differences are mainly due to the current coarse particle distribution and can be diminished by using more particles.

\section{Concluding remarks}

The problem of flow separation at bends with various leg ratios and turning angles has numerically been simulated by the SPH particle method. The obtained steady states are compared to analytical solutions from potential flow theory. For a right-angled bend with different ratios $\left(R_{b}\right)$ of downstream to upstream channel width, the computed free-streamline trajectories agree well with the theory. The difference between the calculated and theoretical flow contraction coefficients is small, with a 1.5 percent maximum relative error. As $R_{b}$ increases, the contraction coefficient first decreases from 0.6 to a minimum value of 0.5 and then increases to a maximum value of 1 . The corresponding limit case $R_{b} \rightarrow \infty$ corresponds to jet flow impinging on a perpendicular wall. For symmetric bends with various turning angles, the computed free streamlines and contraction coefficients match the theoretical results with a maxi- 
mum relative error of 1 percent. One example of flow merging in a branched channel has been simulated and good agreement with theory was found. The current SPH solver appears to be a powerful tool to deal with flow separation problems in channels. The steady solutions were in excellent agreement with theory; the unsteady solutions will be used to estimate impact forces on bends [18].

\section{Acknowledgment}

The first author is grateful to the China Scholarship Council (CSC) for financially supporting his PhD studies. The support in part by the National Basic Research Program of China (No. 2013CB329301) is highly appreciated too.

\section{References}

[1] A. Roshko. A new hodograph for free-streamline theory. NACA Technical Note No. 3168, (1954).

[2] A. Lichtarowicz and E. Markland. Calculation of potential flow with separation in a right-angled elbow with unequal branches. J. Fluid Mech., 17 (1963), 596-606.

[3] R. R. Mankbadi and S. S. Zaki. Computations of the contraction coefficient of unsymmetrical bends. AIAA J., 24 (1986), $1285-1289$.

[4] S. S. Chu. Separated flow in bends of arbitrary turning angles, using the hodograph method and Kirchhoff's free streamline theory. J. Fluids Engrg., 119 (2003), 438-442.

[5] W. C. Hassenpflug. Free-streamlines. Computers Math. Appl., 36, 1 (1998), 69-129.

[6] W. C. Hassenpflug. Branched channel free-streamlines. Comput. Methods Appl. Mech. Engrg., 159 (1998), 329-354.

[7] J. J. Monaghan. Smoothed particle hydrodynamics. Annu. Rev. Astron. Astrophys., 30 (1992), 543-574.

[8] S. Rosswog. Astrophysical smooth particle hydrodynamics. New Astron. Rev., 53 (2009), 78-104.

[9] V. Springel. Smoothed particle hydrodynamics in astrophysics. Annu. Rev. Astron. Astrophys., 48 (2010), 391-430.

[10] L. D. Libersky, A. G. Petschek, T. C. Carney, J. R. Hipp and F. A. Allahdadi. High strain Lagrangian hydrodynamics: A three dimensional SPH code for dynamic material response. J. Comput. Phys., 109 (1993), 67-C75.

[11] J. J. Monaghan. Simulating free surface flows with SPH. J. Comput. Phys., 110 (1994), 399-406.

[12] M. B. Liu and G. R. Liu. Smoothed particle hydrodynamics (SPH): An overview and recent developments. Arch. Comput. Methods. Engrg., 17 (2010), 25-76.

[13] J. J. Monaghan. Smoothed particle hydrodynamics and its diverse applications. Annu. Rev. Fluid Mech., 44, 1 (2012), $323-346$.

[14] D. J. Price. Smoothed particle hydrodynamics and magnetohydrodynamics. J. Comput. Phys., 231 (2012), 759-794.

[15] M. Lastiwka, M. Basa and N. J. Quinlan. Permeable and non-reflecting boundary conditions in SPH. Int. J. Numer. Meth. Fluids, 61 (2009), $709-724$

[16] Q. Hou, L. X. Zhang, A. C. H. Kruisbrink and A. S. Tijsseling. Rapid filling of pipelines with the SPH particle method. Procedia Engrg., 31 (2012), 38-43.

[17] Q. Hou, A. C. H. Kruisbrink, A. S. Tijsseling and A. Keramat. Simulating transient pipe flow with corrective smoothed particle method. BHR Group, 11th International Conference on Pressure Surges, pp. 171-188, Lisbon, Portugal, 2012.

[18] Q. Hou, A. S. Tijsseling and Z. Bozkus. Dynamic force on an elbow caused by a traveling liquid slug. J. Pressure Vessel Technol., ASME, Submitted.

[19] P. J. Reichl, P. Morris, K. Hourigan, M. C. Thompson and S. A. T. Stoneman. Smooth particle hydrodynamics simulation of surface coating. Appl. Math. Model., 22 (1998), 1037-1046.

[20] D. Molteni and A. Colagrossi. A simple procedure to improve the pressure evaluation in hydrodynamic context using the SPH. Comput. Phys. Commun., 180 (2009), 861-872. 
[21] Q. Hou. Simulating unsteady conduit flows with smoothed particle hydrodynamics. PhD thesis, Eindhoven University of Technology, Eindhoven, The Netherlands, 2012. Available from http://repository.tue.n1/733420.

[22] J. P. Morris, P. J. Fox and Y. Zhu. Modelling low Reynolds number incompressible flows using SPH. J. Comput. Phys., (136) 1997, 214-226.

[23] M. Griebel, T. Dornseifer and T. Neunhoeffer. Numerical Simulation in Fluid Dynamics: A Practical Introduction. Philadelphia, SIAM, 1998.

[24] S. Marrone, M. Antuono, A. Colagrossi, G. Colicchio, D. Le Touzé and G. Graziani. $\delta$-SPH model for simulating violent impact flows. Comput. Methods Appl. Mech. Engrg., 200 (2011), 1526-1542.

[25] S. Adami, X. Y. Hu and N. A. Adams. A generalized wall boundary condition for smoothed particle hydrodynamics. J. Comput. Phys., 231 (2012), 7057-7075.

[26] S. J. Cummins and M. Rudman. An SPH projection method. J. Comput. Phys., 152 (1999), 584-607.

[27] X. Liu, H. H. Xu, S. D. Shao, and P. Z. Lin. An improved incompressible SPH model for simulation of wave-structure interaction. Comput. Fluids, 71 (2013), 113-123.

[28] A. C. H. Kruisbrink, F. R. Pearce, T. Yue, A. Cliffe and H. Morvan. SPH concepts for continuous wall and pressure boundaries. The 6th International SPHERIC Workshop Proceedings, pp. 298-304, Hamburg, Germany, 2011.

[29] I. Federico, S. Marrone, A. Colagrossi, F. Aristodemo and P. Veltri. Simulating free-surface channel flows through SPH. In 5th International SPHERIC Workshop, Manchester, UK, 2010.

[30] S. D. Shao and E. Y. M. Lo. Incompressible SPH method for simulating Newtonian and non-Newtonian flows with a free surface. Adv. Water Resour., 26 (2003), 787-800.

[31] D. A. Fulk and D. W. Quinn. An analysis of 1-D smoothed particle hydrodynamics kernels. J. Comput. Phys., 126 (1996), 165-180.

[32] J. J. Monaghan. Smoothed particle hydrodynamic simulations of shear flow. Mon. Not. R. Astron. Soc. 365 (2006), 199-213.

[33] P. A. Thomas and H. M. P. Couchman. Simulating the formation of a cluster of galaxies. Mon. Not. R. Astron. Soc., 257 (1992), $11-31$.

[34] E. S. Lee, C. Moulinec, R. Xu, D. Violeau, D. Laurence and P. Stansby. Comparisons of weakly compressible and truly incompressible algorithms for the SPH mesh free particle method. J. Comput. Phys., 221 (2008), 8417-436.

[35] H. M. P. Couchman, P. A. Thomas and F. R. Pearce. HYDRA: An adaptive-mesh implementation of PPPM-SPH. Astrophys. J., 452 (1995), 797-813.

[36] N. J. Quinlan and M. Lastiwka. Truncation error in mesh-free particle methods. Int. J. Numer. Meth. Engng., 66 (2006), $2064-2085$.

[37] G. L. Vaughan, T. R. Healy, K. R. Bryan, A. D. Sneyd and R. M. Gorman. Completeness, conservation and error in SPH for fluids. Int. J. Numer. Meth. Fluids, 56 (2008), 37-62.

[38] M. Ellero and N. A. Adams. SPH simulations of flow around a periodic array of cylinders confined in a channel. Int. J. Numer. Meth. Engng., 86 (2011), 1027-1040.

[39] W. Peng and D. F. Parker. An ideal fluid jet impinging on an uneven wall. J. Fluid Mech., 333 (1997), $231-255$.

[40] M. Antuono, A. Colagrossi, S. Marrone and D. Molteni. Free-surface flows solved by means of SPH schemes with numerical diffusive terms. Comput. Phys. Commun., 181 (2010), 532-549. 
PREVIOUS PUBLICATIONS IN THIS SERIES:

\begin{tabular}{|c|c|c|c|}
\hline Number & Author(s) & Title & Month \\
\hline $13-29$ & $\begin{array}{l}\text { J.H.M. ten Thije } \\
\text { Boonkkamp } \\
\text { L. Liu } \\
\text { J. van Dijk } \\
\text { K.S.C. Peerenboom }\end{array}$ & $\begin{array}{l}\text { Harmonic complete flux } \\
\text { schemes for conservation } \\
\text { laws with discontinuous } \\
\text { coefficients }\end{array}$ & Nov. 'I3 \\
\hline $13-30$ & $\begin{array}{l}\text { R. Pulch } \\
\text { E.J.W. ter Maten } \\
\text { F. Augustin }\end{array}$ & $\begin{array}{l}\text { Sensitivity analysis of } \\
\text { linear dynamical systems } \\
\text { in uncertainty } \\
\text { quantification }\end{array}$ & Nov. 'I3 \\
\hline I3-3I & $\begin{array}{l}\text { R. Pulch } \\
\text { E.J.W. ter Maten }\end{array}$ & $\begin{array}{l}\text { Stochastic Galerkin } \\
\text { methods and model order } \\
\text { reduction for linear } \\
\text { dynamical systems }\end{array}$ & Nov. 'I3 \\
\hline $13-32$ & $\begin{array}{l}\text { K. Kumar } \\
\text { M. Neuss-Radu } \\
\text { I.S. Pop }\end{array}$ & $\begin{array}{l}\text { Homogenization of a pore } \\
\text { scale model for } \\
\text { precipitation and } \\
\text { dissolution in porous } \\
\text { media }\end{array}$ & Dec. 'I3 \\
\hline I3-33 & $\begin{array}{l}\text { Q. Hou } \\
\text { A.C.H. Kruisbrink } \\
\text { F.R. Pearce } \\
\text { A.S. Tijsseling } \\
\text { T. Yue }\end{array}$ & $\begin{array}{l}\text { Smoothed particle } \\
\text { hydrodynamics } \\
\text { simulations of flow } \\
\text { separation at bends }\end{array}$ & Dec. 'I3 \\
\hline
\end{tabular}

